\title{
Analysis and Research on Capacitive Maize Moisture Sensor
}

\author{
Lixin Zhang ${ }^{1, a}$, Yankun Lan ${ }^{2, b}$, Chuanlu Ren ${ }^{3, c}$ \\ 'Jilin teachers' Institute of Engineering and Technology, Changchun, China \\ ${ }^{2}$ Jilin Branch of China Grain Reserves Corporation, Changchun, China \\ ${ }^{3}$ Jilin Branch of China Grain Reserves Corporation, Changchun, China \\ azlx8015@126.com, ${ }^{b} 303905355 @ q q . c o m,{ }^{c}$ dongnana860901@163.com
}

Keywords: Corn moisture detection Capacitive moisture sensor Mathematical model Equivalent circuit.

Abstract. This paper introduces the structure and principle of the capacitive corn moisture sensor, and focuses on the mathematical model and the equivalent circuit of cylindrical corn moisture sensor, also conductes a preliminary analysis of the factors affecting maize water measurement accuracy, provides a theoretical basis for the design of a cylindrical capacitor corn moisture sensor.

\section{Introduction}

Based on China's grain and oil storage Technical Specifications LS / T1211-2008 requirements, grain storage must be guaranteed before storage under safe moisture, if not, it may cause mildew, which can bring huge economic losses and threaten our food security.To maize, the safe water refers the maximum moisture content of moldy which can not heat the corn in the local environment safely in summer under normal storage conditions, this value is slightly different in different regions of China, In Northeast China the score of corn safe water should not exceed15\%. I n harvest autumn,the water in north China is typically $15-20 \%$, in the northeast and Inner Mongolia region it is generally 20-35\%, significantly higher than the safe moisture level, in this situation, we must take actions to precipitate. Usually the main method used is to use grain dryer to precipitate, makes the corn moisture level reaching the safe level, so the corn moisture sensor is a key parameter of grain dryer to control grain moisture.

There are many measurements of corn moisture sensor, usually such as electrical resistance, capacitance, microwave, infrared, nuclear magnetic resonance method. Since the capacitance method has simple structure, low cost, high sensitivity and good dynamic characteristics, and is suitable for on-line measurement of moisture corn, so it often used in this kind of moisture sensor grain dryer automatic control system.

\section{Principle of capacitive moisture sensor}

According to the composite structure,capacitive moisture sensor is divided into two kinds: flat plate type and cylinder type, the structure is shown in Fig.1 and Fig.2 . Irrespective capacitor plate edge effects, the capacitance of the capacitor is:

$c=\frac{\varepsilon s}{d}$ in it,$\varepsilon$-dielectric constant, $s$-the relative area of the plate, $d$ - the distance between the plates.

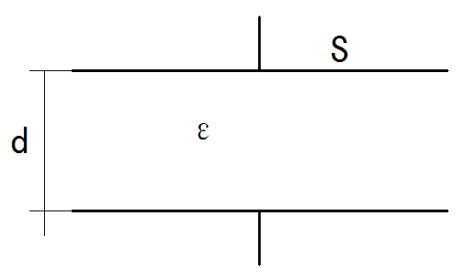

Fig 1. Flatbed capacitive sensor 

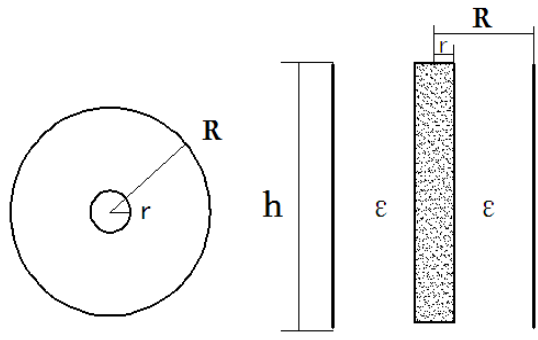

Fig 2. Cylindrical capacitive sensor

Thus, the capacitance measurement is divided into three type : pole-pitch type, variable area type and variable dielectric constant type, and usually the variable dielectric constant measurement method is used to detect objects in the water content. The main component of corn are starch, protein, fat, water and a small amount of minerals and so on, wherein the water comprises a combination water and free water, based on the water is not conductive and free water has the nature of ordinary water,it is the main factor caused the corn moldy and fever, therefore maize water detected generally is refers to the free water, its relative dielectric constant is almost 80 , far higher than the dielectric constant of other substances. The principle of measuring corn moisture capacitance is based on this characteristic.the capacitor plates are filled with corn, due to different corn moisture and different measured capacitance values, treating the circuit and converting the capacitance changes into a change in voltage or frequency, in order to establish a single-valued relationship between water content and characteristics of voltage or frequency.

\section{The mathematical model cylinder Moisture Sensor}

In maize water capacitive sensor system, in order to obtain a larger capacitance value and sensitivity, we usually use cylindrical moisture sensor.Baced on the capacitor structure of Fig. 2, ignoring the capacitor edge effect conditions, when the medium cylindrical capacitive sensor inside the air, the relative dielectric constant is $\varepsilon$, The moisture sensor capacitance $\mathrm{C}$ as shown below.

$$
c=\frac{2 \pi \varepsilon_{0} \varepsilon h}{\ln \frac{R}{r}}
$$

In the above formulas, $\varepsilon_{0}$ is the dielectric constant of vacuum, $h$ is the height of the cylinder, $R$ is the outer radius of the plate cylinder, $r$ is the plate cylinder of radius. When the media is full of corn within the sensor, since the corn is granular objects that exist the air gap inside, so it can be divided the measured medium into the corn and the air in two parts. Under a uniform air gap within the maize, assuming $\varepsilon_{r}$ is the corn relative permittivity within the sensor $h_{1}$ is equivalent height, $\varepsilon$ is relative dielectric constant of air, $h_{2}$ is the equivalent height, then the moisture sensor output capacitance value $\mathrm{C}$ can be shown in formula (1).

$$
c=c_{r}+c_{0}=\frac{2 \pi \varepsilon_{0} \varepsilon_{r} h_{1}}{\ln \frac{R}{r}}+\frac{2 \pi \varepsilon_{0} \varepsilon h_{2}}{\ln \frac{R}{r}}=\frac{2 \pi \varepsilon_{0}}{\ln \frac{R}{r}}\left(h_{1} \varepsilon_{r}+h_{2} \varepsilon\right)
$$

According to formula (1) we can obtaine the mathematical model circuit of cylindrical moisture sensor which is shown in Fig.3, it is the sum of the corn the capacitor dielectric ${ }^{c_{r}}$ and air dielectric capacitor $^{c_{0}}$. After determining the basic dimensions of the moisture sensor, the capacitance value $c_{0}$ is approximately an air dielectric constant, the mathematical relationship between output capacitance value $\mathrm{C}$ and the moisture content of the corn moisture sensor is a function of increasing characteristic, i.e. the higher the moisture content of the corn, the greater the moisture sensor output capacitance value $\mathrm{C}$. 


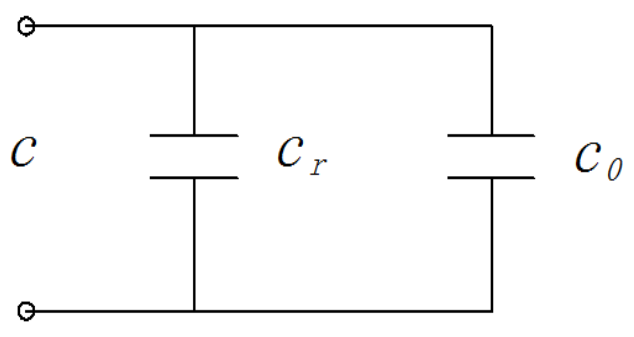

Fig 3. Moisture sensor mathematical model

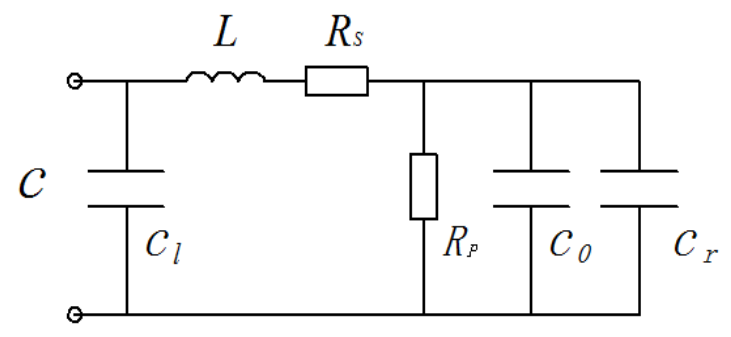

Fig.4 Moisture Sensor equivalent circuit

\section{Factors of cylindrical Moisture Sensor}

Theoretically moisture sensor is based on a mathematical model under ideal conditions. It ignores the many factors. Moisture sensor actual equivalent circuit shown in Fig.4. The sensors take into account all losses capacitive sensors, distributed capacitance and inductance effects.According to the equivalent circuit seen. We use capacitive sensors measure the corn moisture should pay attention to the following factors:

(1)Corn bulk density and air humidity.Equation (1) it is established under ideal conditions. The bulk density and maturity, particle shape of the actual grain corn are related. These factors will affect the equivalent height of $h_{1}$ and $h_{2}$.Formula ignore air dielectric capacitor $c_{0}$ is in the air, it is relatively dry conditions. If the air humidity will directly affect the measurement results, it can even lead to serious results deviate from the actual measurements.

(2)Dielectric loss Sensor.Insulation between the capacitor plates can not really, in the equivalent circuit $R_{P}$ represents a parallel loss resistance. It is the leakage resistance and dielectric loss between

the capacitor plates. $R_{S}$ represents a series loss resistance, which is the lead resistance and plate resistance. These losses at low frequencies greater impact on the measurement results of the sensor.

(3)Inductive effect sensors.Equivalent inductance circuit L represents a sensor, which includes an inductor and a capacitor itself, the external lead inductance. When the circuit is low frequency that it can be ignored, but higher operating frequency can also influence the measurement results of the sensor.

(4)Capacitive effect sensors. The actual capacitive moisture sensor has two wiring, they form a distributed capacitance between lines. $C_{l}$ represents an equivalent capacitance value of the lead circuit, the value is generally only a few picofarads. Size and distance between two lead wire capacitance between the length and around the relevant degree. When the circuit at higher frequencies it will affect the measurement results of the sensor.

(5)Sensor operating frequency.Capacitive moisture sensor generally use the AC excitation source. Its operating frequency selection is directly related to the sensitivity of the moisture sensor. Figure 4 is an equivalent circuit of the moisture sensor can know the existence of a natural resonant frequency of the circuit, typically tens of megahertz. Therefore, the operating frequency of the resonant circuit of the band must be avoided, otherwise the circuit does not work. 


\section{Conclusions}

Based on the cylindrical corn moisture sensor mathematical model and equivalent circuit analysis, which provides a theoretical basis for the design and use of capacitive moisture sensors. In the design of the sensor should pay attention to the structure of rationality, process simplicity, the density of the material between the plates to maintain uniformity, detained or disjointed phenomenon does not occur. Taking fully into account the presence of dielectric loss capacitive moisture sensor, the distributed capacitance and inductance effects in order to select the appropriate operating frequencies and conversion circuit, give it a higher sensitivity, improved noise immunity of the circuit.

\section{References}

[1] Youwen Yu. Sensors Principles and Project Application.Xi'an University of Electronic Science and Technology Press, In Chinese,2014.

[2] Yupeng Tian. Sensor principle.Science Press, In Chinese, 2007.

[3] Baofeng Zai. Grain moisture detection with capacitive sensor.Sensor Technology Press, In Chinese, 2003.

[4] Liming Cai. Mathematical model and the impact factor of the cylindrical capacitive moisture sensor analysis.Analytical Instruments press, In Chinese, 2009. 